\title{
TITLE:
}

\section{Analytic study on the intrinsic zeros of sampled-data systems}

$\operatorname{AUTHOR}(\mathrm{S})$ :

Hagiwara, T

CITATION:

Hagiwara, T. Analytic study on the intrinsic zeros of sampled-data

systems. IEEE Transactions on Automatic Control 1996, 41(2): 261-263

\section{ISSUE DATE:}

1996-02

URL:

http://hdl.handle.net/2433/39967

\section{RIGHT:}

(c) 1996 IEEE. Personal use of this material is permitted. However, permission to reprint/republish this material for advertising or promotional purposes or for creating new collective works for resale or redistribution to servers or lists, or to reuse any copyrighted component of this work in other works must be obtained from the IEEE. 


\section{Analytic Study on the Intrinsic Zeros of Sampled-Data Systems}

Tomomichi Hagiwara

\begin{abstract}
This paper investigates the properties of the mapping from the simple zero $\gamma$ of a scalar continuous-time system to the corresponding zero $\Gamma(T)$ of the sampled-data system that results by its discretization using a zero-order hold, where $T$ is the sampling period. It is shown that $\Gamma(T)$ admits a Taylor expansion with respect to $T$, and that it coincides with that of $\exp (\gamma T)$ at least up to the second-order term, in general, and at least up to the third-order term if the relative degree of the continuoustime system is greater than or equal to two. The result is applied to derive a new stability condition of $\Gamma(T)$ for sufficiently small sampling periods.
\end{abstract}

\section{INTRODUCTION}

It is widely recognized that a zero-order hold is one of the basic elements in the implementation of digital control systems. Thus, it has been of fundamental interest to clarify the properties of the sampled-data system $G_{T}(z)$ obtained by the discretization of the continuous-time system $G(s)$ using a zero-order hold [4], [6], [7], [15]-[17], where $T$ is the sampling period. As is well known, by such discretization, the pole $\lambda$ of $G(s)$ is mapped to the pole $\Lambda(T)=\exp (\lambda T)$ of $G_{T}(z)$. However, the mapping of a zero is not so simple that it is generally impossible to derive a closed-form expression of the zero $\Gamma(T)$ of $G_{T}(z)$ that corresponds to the zero $\gamma$ of $G(s)$ in terms of the parameters of $G(s)$ and $T$. Thus, many studies have been carried out about the zeros of $G_{T}(z)$ [1], [3], [5], [8]-[14].

In this paper, confining ourselves to the case of scalar systems, we show that $\Gamma(T)$ admits a Taylor expansion with respect to $T$ if $\gamma$ is a simple zero of $G(s)$. Furthermore, we show that the expansion coincides with that of $\exp (\gamma T)$ at least up to the second-order term, in general, and at least up to the third-order term if the relative degree of $G(s)$ is greater than or equal to two. The result is applied to derive a new stability condition of $\Gamma(T)$ for sufficiently small $T$. Some comments are also given on the case where $\gamma$ is a multiple zero of $G(s)$

In the following, let $(c, A, b)$ be a minimal realization of $G(s)$ :

$$
G(s)=c(s I-A)^{-1} b
$$

where $A \in \boldsymbol{R}^{n \times n}, b \in \boldsymbol{R}^{n \times 1}, c \in \boldsymbol{R}^{1 \times n}$. Then, it is well known (see, e.g., [6] and [10]) that the zeros of $G(s)$ and $G_{T}(z)$ are, respectively, given by the roots of the polynomials

$$
N(s)=\operatorname{det}\left[\begin{array}{cr}
s I-A & -b \\
c & 0
\end{array}\right]
$$

and

$$
N_{T}(z)=\operatorname{det}\left[\begin{array}{cc}
z I-A_{T} & -b_{T} \\
c & 0
\end{array}\right]
$$

where

$$
A_{T}=\exp (A T), \quad b_{T}=\int_{0}^{T} \exp (A t) b d t
$$

Manuscript received April 5, 1994; revised September 15, 1994.

The author is with the Department of Electrical Engineering, Kyoto University, Yoshida, Sakyo-ku, Kyoto 606-01, Japan.

Publisher Item Identifier S 0018-9286(96)00984-1.

\section{MAIN Results-TAYLOR EXPANSION OF $\Gamma(T)$}

Suppose that $s=\gamma$ is a simple zero of $G(s)$, and let $\mathcal{S}$ be a simplyconnected bounded domain containing $\gamma$ but no other zeros of $G(s)$. The following result is a direct consequence of [10, Theorem 3].

Lemma: There exists $T_{\mathcal{S}}(>0)$ such that for every $T$ with $0<T<T_{\mathcal{S}}, G_{T}(z)$ has exactly one zero in the domain $\exp (\mathcal{S} T):=$ $\{\exp (s T) \mid s \in \mathcal{S}\}(\exists \exp (\gamma T))$.

The above lemma justifies us to say that $G_{T}(z)$ has a zero corresponding to the zero $\gamma$ of $G(s)$ [8]-[10]. Specifically, it is called the intrinsic zero ${ }^{1}$ of $G_{T}(z)$ corresponding to $\gamma$, which we denote by $\Gamma(T)$.

The above lemma means that $\Gamma(T)$ can be approximated by $\exp (\gamma T)$ in some sense, but it is not very clear how close $\Gamma(T)$ is to $\exp (\gamma T)$. On the other hand, it was shown in [13] that $\Gamma(T)$ can be approximated by $1+\gamma T$. The purpose of this paper is to get a more accurate approximation for $\Gamma(T)$. For this purpose, let us suppose that $\Gamma(T)$ admits a power series expansion of the form

$$
\Gamma(T)=1+\gamma T+\eta T^{2}+\xi T^{3}+O\left(T^{4}\right) .
$$

Since $\Gamma(T)$ is a zero of $G_{T}(z)$, it must satisfy

$$
\psi(T):=\operatorname{det}\left[\begin{array}{cc}
\Gamma(T) I-A_{T} & -b_{T} \\
c & 0
\end{array}\right]=0 .
$$

Therefore, our purpose is to find the coefficients $\eta$ and $\xi$ such that the Taylor expansion of $\psi(T)$ with respect to $T$ becomes as close to zero as possible. More specifically, we are to find $\eta$ and $\xi$ such that $\left.(d / d T)^{k} \psi(T)\right|_{T=0}=0(k=0, \cdots, K)$ for as large $K$ as possible.

The following equation is readily obtained as in [6], [8]-[10] irrespective of $\eta$ and $\xi$, using a formula for the derivative of a determinant:

$$
\left.(d / d T)^{k} \psi(T)\right|_{T=0}=0 \quad(k=0, \cdots, n) .
$$

Next, from the condition $\left.(d / d T)^{k} \psi(T)\right|_{T=0}=0$ for $k=n+1$, we obtain

$$
\operatorname{det}\left[\begin{array}{cc}
\gamma I-A & \hat{b}_{\eta} \\
c & 0
\end{array}\right]=0
$$

where $\hat{b}_{\eta}$ is given by

$$
\hat{b}_{\eta}=(\gamma I-A)^{-1}\left(\eta I-A^{2} / 2\right) b-A b / 2 .
$$

Furthermore, from the condition $\left.(d / d T)^{k} \psi(T)\right|_{T=0}=0$ for $k=$ $n+2$, we obtain

$$
\operatorname{det}\left[\begin{array}{cc}
\gamma I-A & \hat{b}_{\eta \xi} \\
c & 0
\end{array}\right]=0
$$

where

$$
\begin{aligned}
\hat{b}_{\eta \xi}= & -A^{2} b / 6+\left(\xi I-A^{3} / 6\right)(\gamma I-A)^{-1} b \\
& -\left[\left(\eta I-A^{2} / 2\right)(\gamma I-A)^{-1}\right]^{2} b \\
& +\left(\eta I-A^{2} / 2\right)(\gamma I-A)^{-1} A b / 2 \\
& +\operatorname{trace}\left(\left(\eta I-A^{2} / 2\right)(\gamma I-A)^{-1}\right) \\
& \cdot\left[\left(\eta I-A^{2} / 2\right)(\gamma I-A)^{-1} b-A b / 2\right] .
\end{aligned}
$$

The conditions (8) and (10), and even higher order conditions, can be derived using essentially the same technique as that employed in the proof of [8, Lemma 1] and [10, Lemma 1] (basically, differentiate

${ }^{1}$ A zero of $G_{T}(z)$ is called an intrinsic zero if it corresponds to a zero of $G(s) . G_{T}(z)$ often has a zero that has no continuous-time counterpart [1], which we call a discretization zero of $G_{T}(z)$. See [8]-[10] for more details. 
the matrix in (6) row by row repeatedly and add and subtract appropriate terms to arrange the results using the Laplace expansion of a determinant). The lengthy derivations are not repeated here.

Since (8) is equivalent to $c(\gamma I-A)^{-1} \hat{b}_{\eta}=0$, we obtain from (9) the following equation for $\eta$ :

$$
c(\gamma I-A)^{-2} b \cdot \eta=c(\gamma I-A)^{-2} A^{2} b / 2+c(\gamma I-A)^{-1} A b / 2 .
$$

Now, by the assumption that $\gamma$ is a simple zero of $G(s)$, we have

$$
c(\gamma I-A)^{-2} b=-G^{\prime}(\gamma) \neq 0
$$

where $G^{\prime}(s)$ denotes $(d / d s) G(s)$. Therefore, $\eta$ can be obtained as

$$
\begin{aligned}
\eta & =\frac{c(\gamma I-A)^{-2} A^{2} b+c(\gamma I-A)^{-1} A b}{2 c(\gamma I-A)^{-2} b} \\
& =\frac{\gamma c(\gamma I-A)^{-2} A b}{2 c(\gamma I-A)^{-2} b} \\
& =\gamma^{2} \cdot 2
\end{aligned}
$$

where we added $\gamma c(\gamma I-A)^{-1} b=0$ to the numerator to get the last expression.

Substituting the above equation into $(11), \hat{b}_{\eta \xi}$ reduces to $\hat{b}_{\xi}$, where

$$
\begin{aligned}
\hat{b}_{\xi}= & -A^{2} b / 6+\left(\xi I-A^{3} / 6\right)(\gamma I-A)^{-1} b \\
& -\gamma(\gamma I+A) b / 4+\gamma \operatorname{trace}(\gamma I+A) b / 4 .
\end{aligned}
$$

Then, since (10) is equivalent to $c(\gamma I-A)^{-1} \hat{b}_{\xi}=0$, we obtain from (15) and $c(\gamma I-A)^{-1} b=0$ the following equation for $\xi$ :

$$
c(\gamma I-A)^{-2} b \cdot \xi=c(\gamma I-A)^{-1} \hat{b}
$$

where

$$
\begin{aligned}
\hat{b} & =A^{2} b / 6+(\gamma I-A)^{-1} A^{3} b / 6+\gamma A b / 4 \\
& =\gamma(\gamma I-A)^{-1} A^{2} b / 6+\gamma A b / 4 \\
& =\gamma A b / 12+\gamma^{2}(\gamma I-A)^{-1} A b / 6 .
\end{aligned}
$$

Therefore, $\xi$ can be obtained as

$$
\xi=\frac{\gamma c(\gamma I-A)^{-1} A b / 12+\gamma^{2} c(\gamma I-A)^{-2} A b / 6}{c(\gamma I-A)^{-2} b} .
$$

Here, since $s G(s)=c(s I-A)^{-1} A b+c b$, we have $G(s)+s G^{\prime}(s)=$ $-c(s I-A)^{-2} A b$. From these equations and from $G(\gamma)=0$, we obtain $c(\gamma I-A)^{-1} A b=-c b$ and $c(\gamma I-A)^{-2} A b=-\gamma G^{\prime}(\gamma)$. Substituting these and (13) into (18), we obtain

$$
\xi=\gamma^{3} / 6+\gamma c b / 12 G^{\prime}(\gamma)
$$

Continuing the above manner, it is easily seen that we can derive the Taylor expansion ${ }^{2}$ of $\Gamma(T)$ which justifies (5). To summarize the above arguments, we have shown that

$$
\Gamma(T)=1+\gamma T+\frac{\gamma^{2}}{2} T^{2}+\left(\frac{\gamma^{3}}{6}+\frac{\gamma c b}{12 G^{\prime}(\gamma)}\right) T^{3}+O\left(T^{4}\right) .
$$

Noting that $c b=0$ if the relative degree of $G(s)$ is greater than or equal to two, we obtain the following theorem.

Theorem 1: Suppose that $\gamma$ is a simple zero of $G(s)$. Then, $\Gamma(T)$ admits a Taylor expansion with respect to $T$, and it coincides with that of $\exp (\gamma T)$ at least up to the second-order term. In particular, if the relative degree of $G(s)$ is greater than or equal to two, they coincide at least up to the third-order term.

\footnotetext{
${ }^{2}$ The expansion is possible in principle, but to express its coefficients in an explicit compact form seems nontrivial.
}

Remark 1: Even if the relative degree of $G(s)$ is one, the thirdorder terms still coincide if $\gamma=0$. Actually, $\Gamma(T)=1$ for any $T(>0)$ if $\gamma=0$, regardless of the relative degree of $G(s)$ (see, e.g., [6]), and thus $\Gamma(T)=\exp (\gamma T)$ is always true if $\gamma=0$.

Remark 2: If the relative degree of $G(s)$ is greater than or equal to two, $\Gamma(T)=\exp (\gamma T)$ can be the case. For example, for

$$
G(s)=\frac{s-\gamma}{(s-p)(s-q)(s-2 \gamma)} \quad(\gamma=(p+q) / 2)
$$

the zeros of $G_{T}(z)$ are given by $\pm \exp (\gamma T)$.

\section{Application to the Stability Condition of $\Gamma(T)$}

In this section, we study the stability of $\Gamma(T)$, where it is said to be stable if it lies inside the unit circle. From the lemma, the following result is immediate [8]-[10].

Corollary: For any zero $\gamma$ of $G(s),|\Gamma(T)|<1$ (respectively, $|\Gamma(T)|>1$ ) for sufficiently small $T$ if $\Re(\gamma)<0$ (respectively $\Re(\gamma)>0$ ).

From this result, we can check the stability of $\Gamma(T)$ if the zero $\gamma$ of $G(s)$ is not on the imaginary axis. However, if it is on the imaginary axis, the lemma is not helpful to examine stability of the corresponding zero $\Gamma(T)$, because $\exp (S T)$ necessarily contains the points both inside and outside the unit circle. From this difficulty, no stability condition of $\Gamma(T)$ has been obtained for the case of $\Re(\gamma)=0$ (except the special case of $\gamma=0$ as described in remark 1). In the following, we give a stability condition for such a case using the results of the preceding section.

Now, suppose that $\gamma=j \beta(\neq 0)$ so that $\gamma$ is on the imaginary axis. Then, from (5) and (14), we obtain

$$
\begin{aligned}
\Gamma(T)= & \left(1-\frac{\beta^{2}}{2} T^{2}+\sigma T^{3}+O\left(T^{4}\right)\right) \\
& +j\left(\beta T+\omega T^{3}+O\left(T^{4}\right)\right)
\end{aligned}
$$

where

$$
\sigma:=\Re(\xi), \quad \omega:=\Im(\xi) .
$$

Therefore, we obtain

$$
\begin{aligned}
|\Gamma(T)|^{2}= & \left(1-\frac{\beta^{2}}{2} T^{2}+\sigma T^{3}\right)^{2} \\
& +\left(\beta T+\omega T^{3}\right)^{2}+O\left(T^{4}\right) \\
= & 1+2 \sigma T^{3}+O\left(T^{4}\right) .
\end{aligned}
$$

From this equation, we can conclude that $|\Gamma(T)|<1$ (respectively, $|\Gamma(T)|>1$ ) for sufficiently small $T$ if $\sigma<0$ (respectively, $\sigma>0$ ). Here, from (19) and $\gamma=j \beta$, we have

$$
\sigma=\Re(\xi)=\Re\left(\gamma c b / 12 G^{\prime}(\gamma)\right)
$$

In the following, we assume that the relative degree of $G(s)$ is one so that $c b \neq 0$. Then, $|\Gamma(T)|<1$ (respectively, $|\Gamma(T)|>1$ ) if $c b$ and $\Re\left(\gamma / G^{\prime}(\gamma)\right)$ have opposite signs (respectively, the same sign). Here, let us rewrite $G(s)$ in the form

$$
G(s)=\tilde{N}(s)\left(s^{2}-\gamma^{2}\right) / D(s)
$$

where $\tilde{N}(s)$ and $D(s)$ are coprime polynomials. Then, we can easily verify that

$$
\gamma / G^{\prime}(\gamma)=D(\gamma) / 2 \tilde{N}(\gamma)
$$

Next, let us rewrite $1 / G(s)$ in the form

$$
\frac{1}{G(s)}=\left(p_{1} s+p_{0}\right)+\frac{q(s)}{\tilde{N}(s)}+\frac{r_{1} s+r_{0}}{s^{2}-\gamma^{2}}
$$


TABLE I

$|\Gamma(T)|$ FOR EXAMPLE

\begin{tabular}{l|l}
\hline$T$ & $|\Gamma(T)|$ for $G_{1}(s)$ \\
\hline 0.01 & 1.0000000417 \\
0.1 & 1.0000413 \\
0.5 & 1.00407 \\
1 & 0.9987 \\
\hline
\end{tabular}

\begin{tabular}{c|l}
\hline$T$ & $|\Gamma(T)|$ for $G_{2}(s)$ \\
\hline 0.01 & 0.9999999583 \\
0.1 & 0.9999578 \\
0.5 & 0.99323 \\
1 & 0.9119 \\
\hline
\end{tabular}

where $q(s)$ is an appropriate polynomial whose degree is less than that of $\tilde{N}(s)$. Then, we can easily show that $c b=1 / p_{1}$. Furthermore, substituting (26) into (28), multiplying the both sides by $s^{2}-\gamma^{2}$, and letting $s=\gamma=j \beta$, we readily obtain $\Re(D(\gamma) / \tilde{N}(\gamma))=r_{0}$.

Combining the above arguments, we are led to the following stability condition of $\Gamma(T)$.

Theorem 2: Suppose that the relative degree of $G(s)$ is one and let $\gamma(\neq 0)$ be a simple zero of $G(s)$ on the imaginary axis. Then, the corresponding zero $\Gamma(T)$ of $G_{T}(z)$ satisfies $|\Gamma(T)|<1$ (respectively, $|\Gamma(T)|>1$ ) for sufficiently small $T$ if $p_{1}$ and $r_{0}$ have opposite signs (respectively, the same sign), where $p_{1}$ and $r_{0}$ are given by (28).

We study simple examples to illustrate the above theorem.

Example: For the stable minimum phase systems

$$
\begin{aligned}
& G_{1}(s)=\frac{(s+1)\left(s^{2}+4\right)}{s^{4}+3 s^{3}+10 s^{2}+16 s+13} \\
& G_{2}(s)=\frac{(s+1)\left(s^{2}+4\right)}{s^{4}+3 s^{3}+10 s^{2}+14 s+11}
\end{aligned}
$$

we have

$$
\begin{aligned}
& \frac{1}{G_{1}(s)}=(s+2)+\frac{1}{s+1}+\frac{3 s+1}{s^{2}+4} \\
& \frac{1}{G_{2}(s)}=(s+2)+\frac{1}{s+1}+\frac{3 s-1}{s^{2}+4} .
\end{aligned}
$$

Therefore from Theorem 2 , we can conclude that for sufficiently small $T$, the $\Gamma(T)$ corresponding to $\gamma=2 j$ lies outside the unit circle for $G_{1}(s)$ and inside the unit circle for $G_{2}(s)$. This is demonstrated in Table I.

\section{Comments on the CASe Where $\gamma$ Is a Multiple Zero}

When $\gamma$ is a multiple zero of $G(s)$, it is easy to see that (8) becomes indefinite with respect to $\eta$ and that (10) reduces to the quadratic equation for only $\eta$ (i.e., $\xi$ vanishes in the equation) given by

$$
G^{\prime \prime}(\gamma)\left(\eta-\gamma^{2} / 2\right)^{2} / 2-\gamma c b / 12=0
$$

where $G^{\prime \prime}(s):=(d / d s)^{2} G(s)$. Therefore, if $\gamma$ is a zero with degree two so that $G^{\prime \prime}(\gamma) \neq 0$, then we can obtain two values of $\eta$ from the above equation, each of which corresponds to one of the two "branches" of $\Gamma(T)$

However, if the degree of $\gamma$ as a zero of $G(s)$ is greater than two so that $G^{\prime \prime}(\gamma)=0$, and if the relative degree of $G(s)$ is one so that $c b \neq 0$, then (33) admits no solution $\eta$ unless $\gamma=0$. This is because the expansion of $\Gamma(T)$ in (5) is not always adequate when $\gamma$ is a multiple zero; the branches of $\Gamma(T)$ do not admit Taylor expansions, in general. This is not surprising in view of the theory of algebraic functions [2]; the expansion of $\Gamma(T)$ would require fractional power of $T$, in general, if $\gamma$ is a multiple zero.

\section{CONCLUSION}

The properties of the zero $\Gamma(T)$ of $G_{T}(z)$ corresponding to the zero $\gamma$ of $G(s)$ are investigated and are applied to derive a new stability condition of $\Gamma(T)$ for sufficiently small $T$.

\section{ACKNOWLEDGMENT}

The author is indebted to Prof. Y. Hayakawa for his motivation to the study through discussions.

\section{REFERENCES}

[1] K. J. Åström, P. Hagander, and J. Sternby, "Zeros of sampled systems," Automatica, vol 20 , no. 1, pp. 31-38, 1984.

[2] G. A. Bliss, Algebraic Functions, New York: Dover, 1966.

[3] Y. Fu and G. A. Dumont, "Choice of sampling to ensure minimum-phase behavior," IEEE Trans. Automat. Contr., vol. 34, no. 5, pp. 560-563, 1989.

[4] J. W. Grizzle and M. H. Shor, "Sampling, infinite zeros and decoupling of linear systems," Automatica, vol. 24, no. 3, pp. 387-396, 1988.

[5] P. Hagander, "Comments on "Conditions for stable zeros of sampled systems," IEEE Trans. Automat. Contr., vol. 38, no. 5, pp. 830-831, 1993.

[6] T. Hagiwara and M. Araki, "On preservation of strong stabilizability under sampling," IEEE Trans. Automat. Contr., vol. AC-33, no. 11, pp. 1080-1082, 1988

[7] — "Controllability indices of sampled-data systems," Int. J. Syst. Sci., vol. 19, no. 12, pp. 2449-2457, 1988

[8] _ - "Properties of limiting zeros of sampled-data systems," Trans. Inst. Elec. Engineers Japan, vol. 110-C, no. 4, pp. 235-244, 1990.

[9] _ _Limiting properties of zeros of sampled-data systems," in Communication, Control and Signal Processing, in Proc. 1990 Bilkent Int. Conf. New Trends Communication, Contr., Signal Processing, E. Arikan, Ed. New York: Elsevier, 1990, pp. 998-1004.

[10] T. Hagiwara, T. Yuasa, and M. Araki, "Stability of the limiting zeros of sampled-data systems with zero- and first-order holds," Int. J. Contr. vol. 58, no. 6, pp. 1325-1346, 1993.

[11] S. Hara, H. Katori, and R. Kondo, "The relationship between real poles and real zeros in SISO sampled data systems," IEEE Trans. Automat. Contr., vol. 34, no. 6, pp. 632-635, 1989.

[12] S. Hara, R. Kondo, and H. Katori, "Properties of zeros in digital control systems with computational time delay," Int. J. Contr., vol. 49, no. 2 , pp. 493-511, 1989.

[13] Y. Hayakawa, S. Hosoe, and M. Ito, "On the limiting zeros of sampled multivariable systems," Syst. Contr. Lett., vol. 2, pp. 292-300, 1983.

[14] M. Ishitobi, "Stable zeros of sampled low-pass systems," Int. J. Contr., vol. 57 , no. 6, pp. 1485-1498, 1993.

[15] R. E. Kalman, Y. C. Ho, and K. S. Narendra, "Controllability of linear dynamical systems," Contributions Differential Equations, vol. 1, no. 2, pp. $189-213,1962$

[16] M. Kimura, "Preservation of stabilizability of a continuous timeinvariant linear system after discretization," Int. J. Syst. Sci., vol. 21 , no. 1, pp. $65-91,1990$.

[17] Ü. Özgüner and E. J. Davison, "Sampling and decentralized fixed modes," in Proc. ACC, 1985, pp. 257-262. 